\title{
Physics with relativistic heavy-ion collisions
}

\author{
Benjamin Dönigus $^{\mathrm{a}}$ \\ Institut für Kernphysik, Goethe-Universität Frankfurt, Max-von-Laue-Straße 1, 60438 Frankfurt, Germany
}

\begin{abstract}
An overview is given on the experimental study of physics with relativistic heavy-ion collisions, with emphasis on recent measurements at the Large Hadron Collider (LHC) and the Relativistic Heavy Ion Collider (RHIC). The focus here is laid on $\mathrm{p}-\mathrm{Pb}$ collisions at the $\mathrm{LHC}$ and the corresponding $\mathrm{d}-\mathrm{Au}$ measurements at RHIC. The topics touched are "collectivity and approach to equilibrium", "high $p_{\mathrm{T}}$ and jets", "heavy flavour and electroweak bosons" and "search for exotic objects".
\end{abstract}

\section{Introduction}

Relativistic heavy-ion physics is usually (especially from the outside of the field) seen as the search for the quarkgluon plasma, a droplet of deconfined matter formed in the very high temperatures of the mentioned collisions which is one of the sequences in the time-evolution of the early universe. It is the main aim of the relativistic heavy-ion physics to create the quark-gluon plasma and to study its properties. To understand these properties, control measurements in $\mathrm{pp}$ and $\mathrm{p}-\mathrm{Pb}$ collisions have to be done. These control measurements provide the largest overlap between the heavy-ion and the high-energy cosmic ray community. So this write-up concentrates on the recent highlights in the area of $\mathrm{p}-\mathrm{Pb}$ collisions at the LHC and the corresponding d-Au measurements at RHIC. Some results in connection to these highlights from $\mathrm{Pb}-\mathrm{Pb}$ and $\mathrm{Au}-\mathrm{Au}$ collisions are also given.

The traditional idea of doing $\mathrm{p}-\mathrm{Pb}$ and $\mathrm{d}-\mathrm{Au}$ collisions is to provide a reference for the $\mathrm{Pb}-\mathrm{Pb}$ and $\mathrm{Au}-\mathrm{Au}$ collisions, in order to investigate cold nuclear matter (initial state) effects. However, the data taken there turned out to be very interesting in itself and raised some questions:

- Do we observe collective effects in a small system such as $\mathrm{p}-\mathrm{Pb}$ collisions? Is there hot matter created in local thermal equilibrium?

- Do we observe an enhancement of high- $p_{\mathrm{T}}$ particles with respect to pp collisions while we see a suppression in $\mathrm{Pb}-\mathrm{Pb}$ collisions?

- What can we learn from heavy flavour and electroweak bosons?

\section{Collectivity and approach to equilibrium}

To answer the questions raised before it is important to distinguish between:

- a system of individual particles and

- a medium in which individual degrees of freedom do not matter anymore and we can apply thermodynamic concepts.

\footnotetext{
${ }^{a}$ e-mail: benjamin.doenigus@cern.ch
}

Thermodynamic concepts are typically used to describe systems with large number of particles $\left(>10^{4}\right)$ in local thermal equilibrium. Typical numbers of produced particles per pseudo-rapidity in collisions at the LHC are:

- in central (0-5\%) Pb-Pb collisions (LHC): $\mathrm{d} N_{\mathrm{ch}} / \mathrm{d} \eta \approx$ 1600 [1], and

- in high multiplicity $(0-5 \%) \mathrm{p}-\mathrm{Pb}$ collisions ( $\mathrm{LHC})$ : $\mathrm{d} N_{\mathrm{ch}} / \mathrm{d} \eta \approx 45$ [2], and

- in minimum bias pp collisions (LHC): $\mathrm{d} N_{\mathrm{ch}} / \mathrm{d} \eta \approx$ $6[3]$.

The lifetime of the created system (medium) must be long enough and the mean free path of the particles in the medium must be short enough, so that equilibrium can be established by several (simulations show about 3-6 are necessary) interactions between its constituents [4].

Two models are usually used to describe collective phenomena connected to heavy-ion physics, they are both quite successful in describing the data:

- hydro models describing flow effects in $\mathrm{Pb}-\mathrm{Pb}$ support the idea of matter in local thermal (kinetic) equilibrium.

- thermal models describing hadron yields in $\mathrm{Pb}-\mathrm{Pb}$ support the idea of matter in local thermal (chemical) equilibrium.

So the big question which is being raised here is: Do we find the forms of equilibrium in smaller systems such as $\mathrm{p}-\mathrm{Pb}$ ?

\subsection{Hydro models, flow and kinetic equilibrium}

In heavy-ion collisions one usually distinguishes between two types of flow in the expanding and cooling down phase of the fireball [5]:

- Isotropic (radial) flow, the isotropic "push" every particle gets, which can be described in a simplified hydro model with a common velocity $\beta$ and kinetic temperature $T_{\text {kin }}$, and

- Anisotropic (elliptic) flow, the flow of particles created by the spatial anisotropy (in eccentric collisions) which transforms into a pressure gradient which pushes particles stronger into one direction than into another.

This is an Open Access article distributed under the terms of the Creative Commons Attribution License 4.0, which permits unrestricted use, distribution, and reproduction in any medium, provided the original work is properly cited. 
(d) $\mathrm{CMS} \mathrm{N} \geq 110,1.0 \mathrm{GeV} / \mathrm{c}<\mathrm{p}_{\mathrm{T}}<3.0 \mathrm{GeV} / \mathrm{c}$

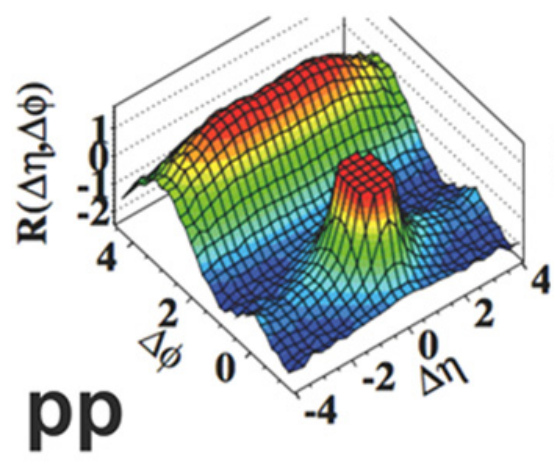

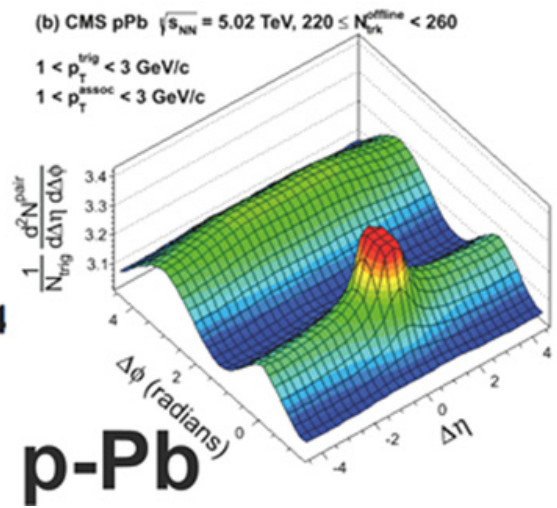

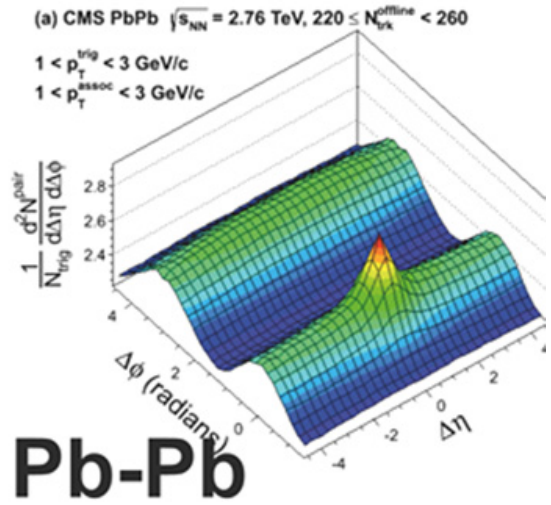

Figure 1. Angular two-particle correlations of inclusive particles for pp (left), $\mathrm{p}-\mathrm{Pb}$ (middle) and $\mathrm{Pb}-\mathrm{Pb}$ (right) measured with $\mathrm{CMS}$ [6,7]
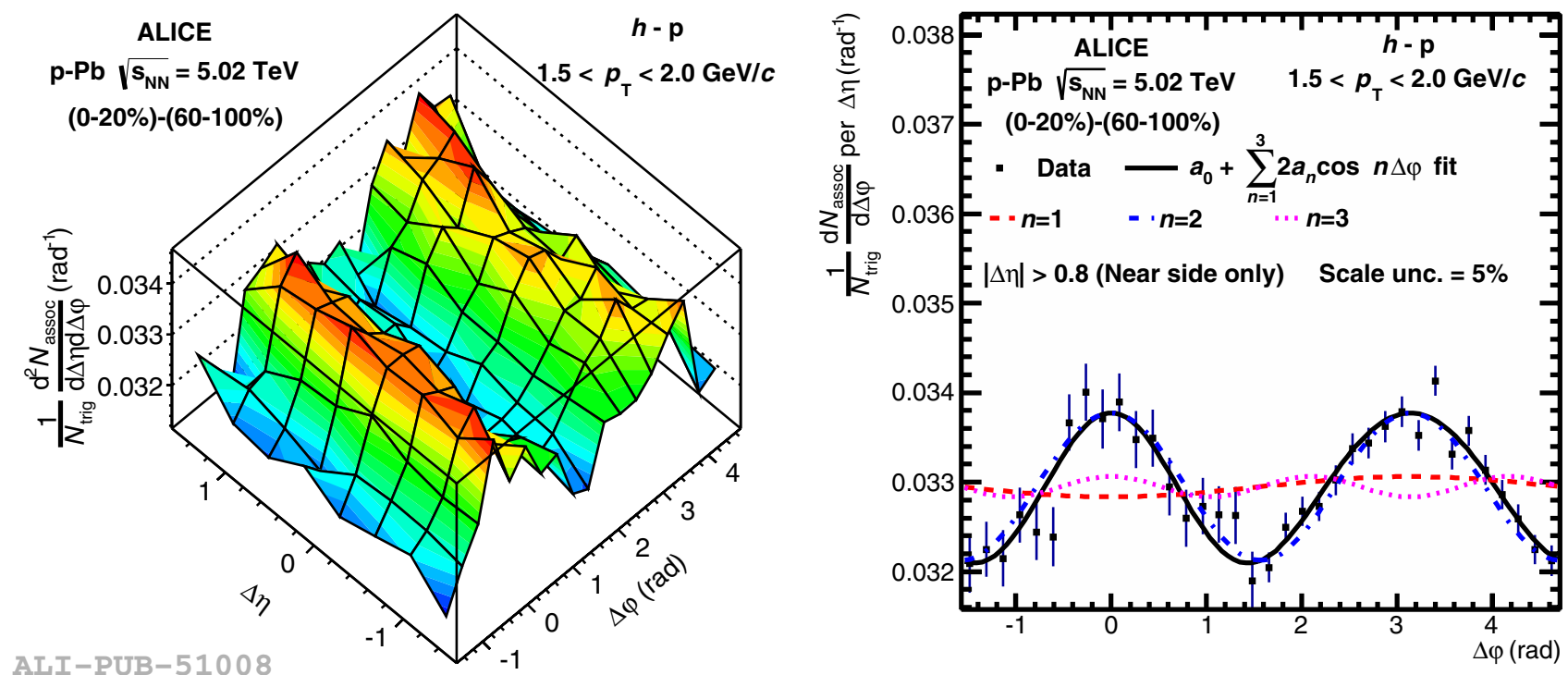

Figure 2. Left panel: associated yield per trigger particle as a function of $\Delta \varphi$ and $\Delta \eta$ for hadron-proton correlations for $1.5<p_{\mathrm{T}}<$ $2 \mathrm{GeV} / c$ for the $0-20 \%$ event class where the corresponding correlation from the $60-100 \%$ event class has been subtracted. Right panel: projection of the left panel correlations to $\Delta \varphi$ averaged over $0.8<|\Delta \eta|<1.6$ on the near side and $|\Delta \eta|<1.6$ on the away side. The figure contains only statistical uncertainties and systematic uncertainties are mostly correlated and are less than 5\% (from [8]).

The aforementioned anisotropic expansion can be decomposed following its Fourier expansion:

$E \frac{d^{3} N}{d^{3} p}=\frac{1}{2 \pi} \frac{d^{2} N}{p_{\mathrm{T}} d p_{\mathrm{T}} d y}\left(1+\sum_{n=1}^{\infty} 2 v_{n} \cos \left(n\left(\phi-\psi_{\mathrm{rp}}\right)\right)\right)$,

where the $v_{n}=\left\langle\cos \left[n\left(\phi_{i}-\psi_{\mathrm{rp}}\right)\right]\right\rangle$ coefficients are used for a quantitative characterization of the event anisotropy, and the angle brackets mean an average over all particles in all events. $v_{1}$ is typically referred to as directed flow, and $v_{2}$ as elliptic flow. The different coefficients are determined for instance with 2-particle correlations. For instance one can measure angular correlations of two particles as shown in Fig. 1. When one subtracts the angular correlations of a peripheral event class from a more central class one can see a double ridge structure which is left-over after subtraction (see Fig. 2). From this double ridge structure one can get the elliptic flow, which is visualised in Fig. 3 for inclusive (charged) hadrons, pions, kaons and protons. The different

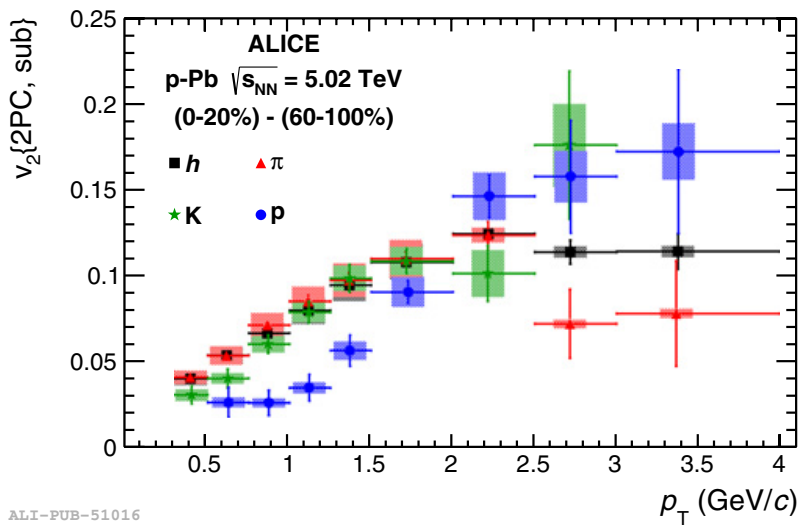

Figure 3. Elliptic flow $v_{2}$, measured for hadrons, pions, kaons and protons in $\mathrm{p}-\mathrm{Pb}$ collisions from ALICE [8].

particles show the same pattern as observed in heavy-ion collisions (see for instance [9]), namely a mass ordering. A possible way to understand this behaviour can be attributed to come from initial (fluctuation) hotspots, which are 


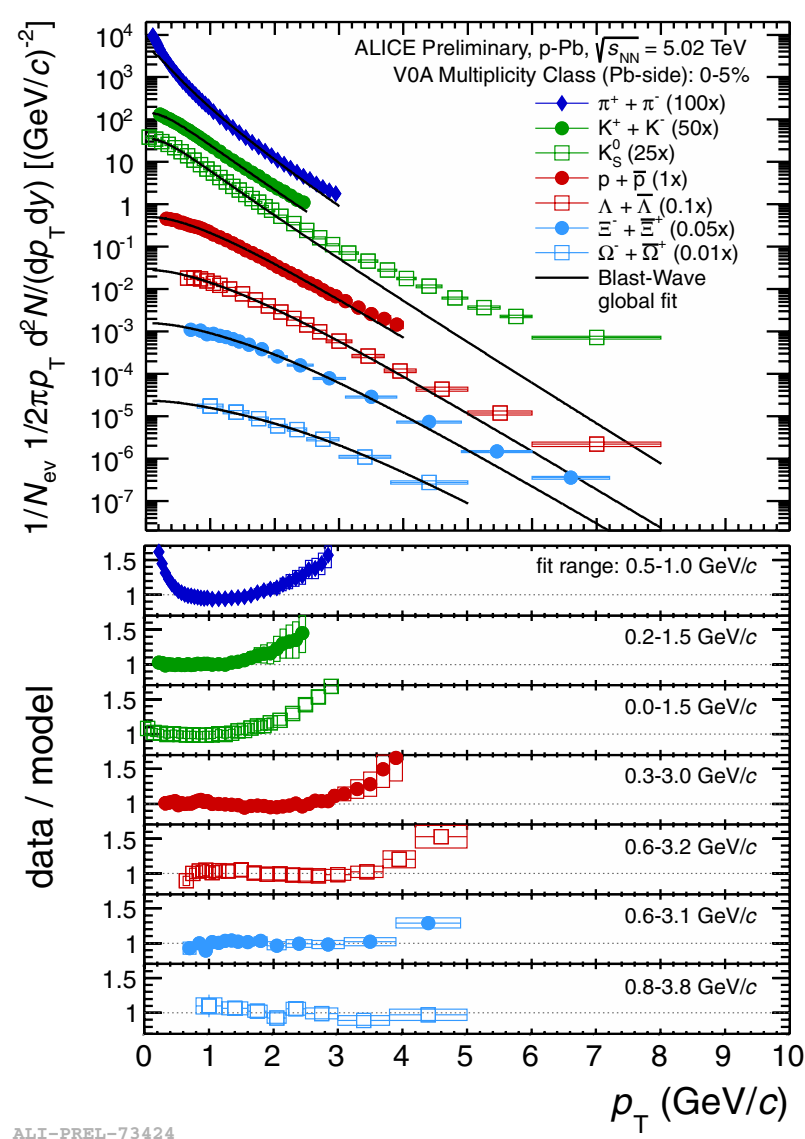

Figure 4. $p_{\mathrm{T}}$ spectra of pions, kaons, protons, Lambda, $\mathrm{Xi}$ and Omega. Fitted altogether with a blast-wave function and the comparison between the fit and the data.

transformed into spatial anisotropies that are then evolving into pressure anisotropies as discussed before.

This means we observe a kind of anisotropic flow in $\mathrm{p}-\mathrm{Pb}$ collisions. What about radial flow? For this we have to look at $p_{\mathrm{T}}$ spectra of particles as displayed in Fig. 4 for pions, kaons, protons, Lambdas, $\mathrm{Xi}$ and Omegas.

A combined blast-wave fit to the data (simplified hydro model, with the two parameters kinetic freezeout temperature $T_{\text {kin }}$ and transverse expansion velocity $\beta$ ) gives a reasonable description, see comparison between data and fit in Fig. 4. In Fig. 5 the $T_{\text {kin }}$ and $\beta$ values for different systems and different multiplicity classes are shown. The $T_{\text {kin }}$ and $\beta$ values for $\mathrm{p}-\mathrm{Pb}$ and $\mathrm{Pb}-\mathrm{Pb}$ data follow the same trend, which is consistent with a collective expansion [11]. Nevertheless, also PYTHIA 8 with color reconnection shows a similar trend (without any hydrodynamic flow). This means other effects can mimic flow-like patterns, and also pp data shows a similar trend as $\mathrm{p}-\mathrm{Pb}$ and $\mathrm{Pb}-\mathrm{Pb}$. One should admit that hydrodynamic models (EPOS, Krakow) show a better agreement than QCD inspired models (DPMJET) [2].

Another interesting fact is that even nuclei show this behaviour and their $p_{\mathrm{T}}$ shape is well described with a simple hydro model, namely by blast-wave fits. Deuterons and anti-deuterons are produced nearly equally in $\mathrm{p}-\mathrm{Pb}$ collisions at the LHC [10].

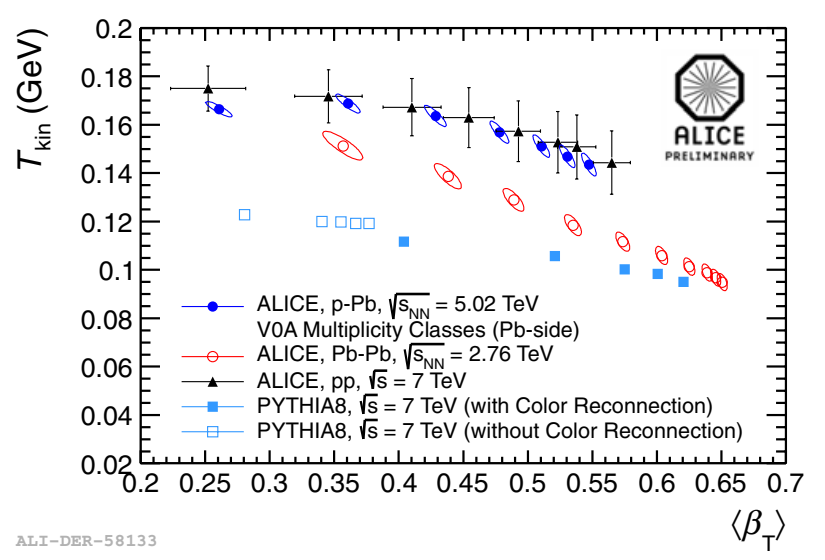

Figure 5. Result of global blast-wave fits, for different systems and compared to PYTHIA simulations with and without the option of colour reconnection, for details see [2].

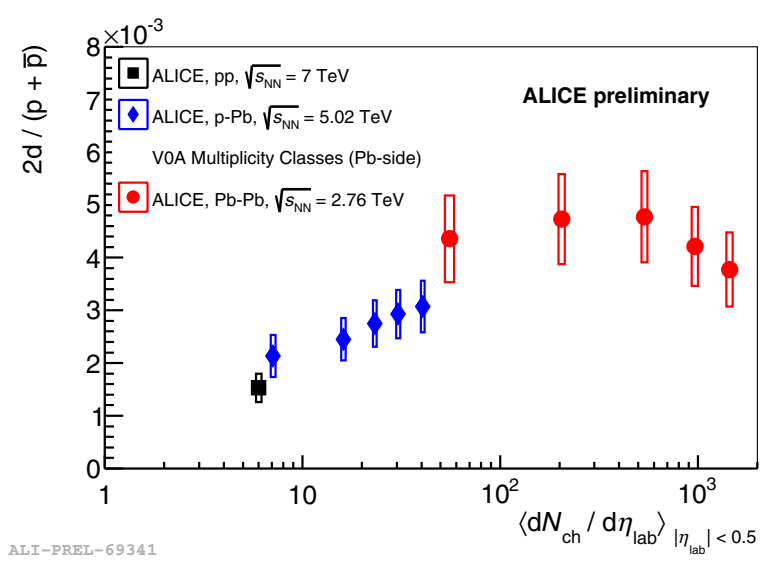

Figure 6. Deuteron-to-proton ratio vs. the event multiplicity, from $\mathrm{pp}$ (black), to $\mathrm{p}-\mathrm{Pb}$ (blue) and $\mathrm{Pb}-\mathrm{Pb}$ (red).

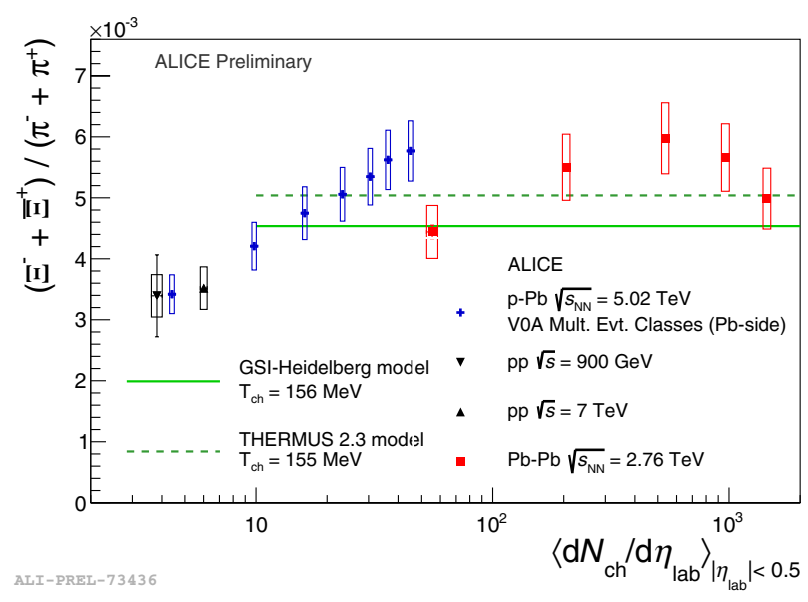

Figure 7. Xi-to-pion ratio over the event multiplicity for three different collision systems. The lines indicate the different predictions from thermal models [12].

\subsection{Particle yields and chemical equilibrium}

If one extracts the production yield in multiplicity classes of different particle species using their $p_{\mathrm{T}}$ spectra (in the given multiplicity or centrality class) one can produce ratios of these yields for a given multiplicity. This is shownin Fig. 6 for the deuteron-to-proton ratio. It shows 


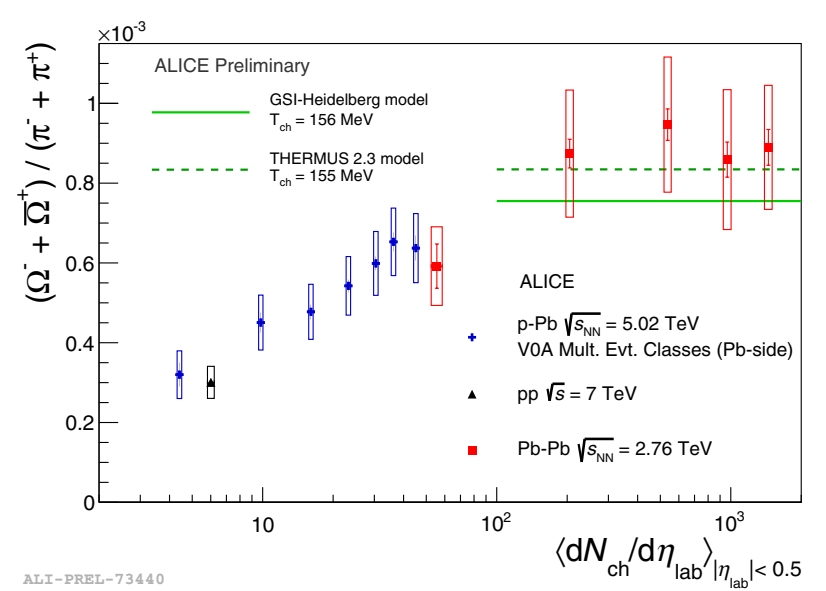

Figure 8. Omega-to-pion ratio vs. the event multiplicity for three different collision systems. The lines indicate the different predictions from thermal models [12].

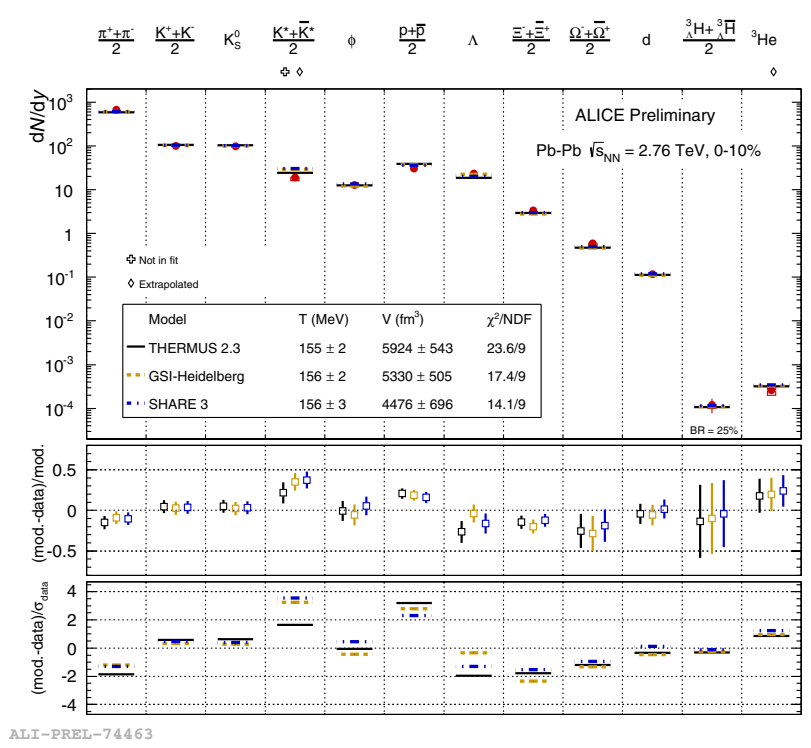

Figure 9. Thermal model fit of ALICE data as shown by M. Floris at QM2014 [13].

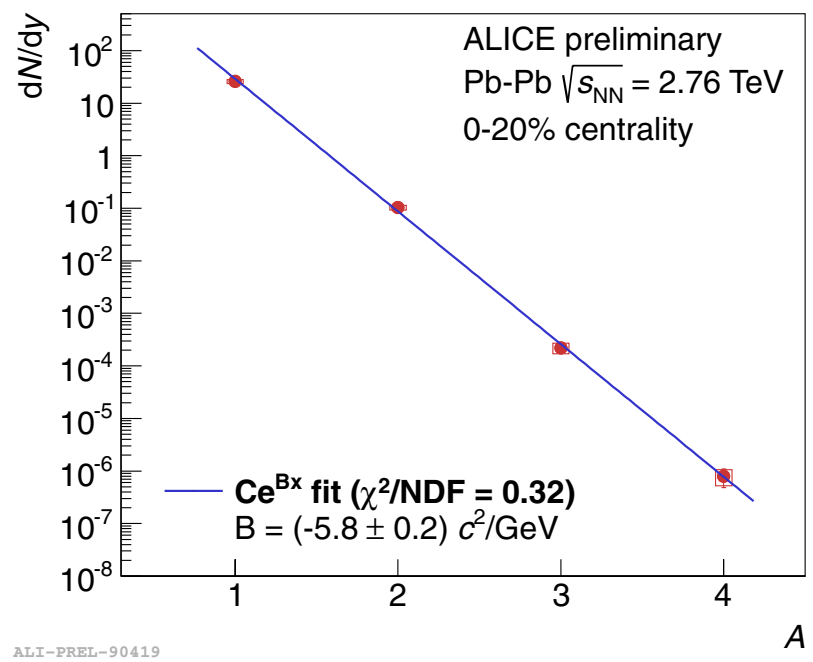

Figure 10. Preliminary yields of protons, deuterons, ${ }^{3} \mathrm{He}$ and anti- ${ }^{4} \mathrm{He}$ measured by ALICE as function of mass number A. The line indicates an exponential fit.

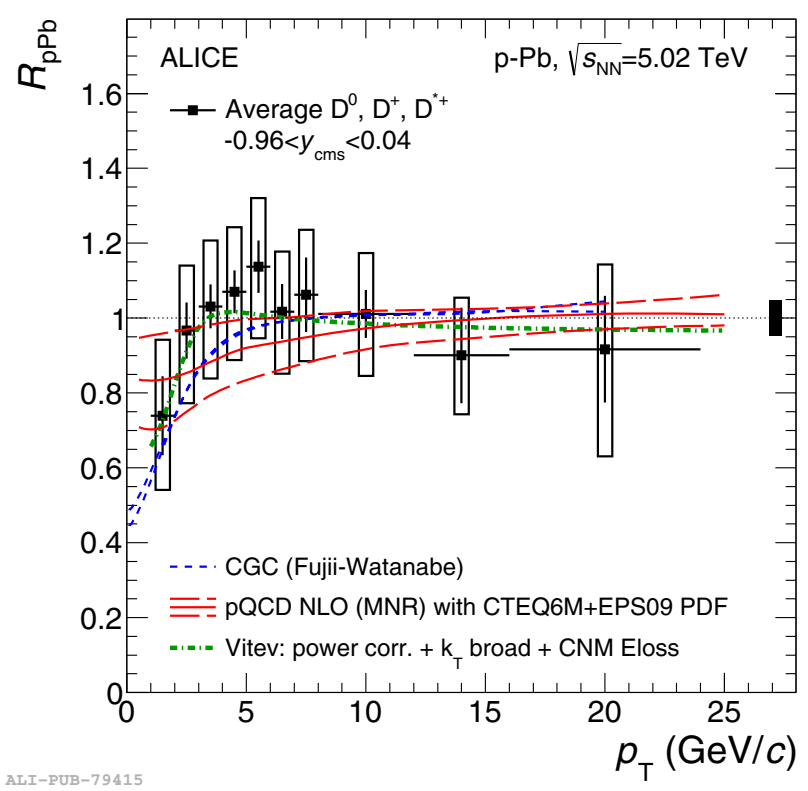

Figure 11. Nuclear modification factor $R_{p A}$ of the average of different $\mathrm{D}$ mesons as measured by the ALICE Collaboration, taken from [21]

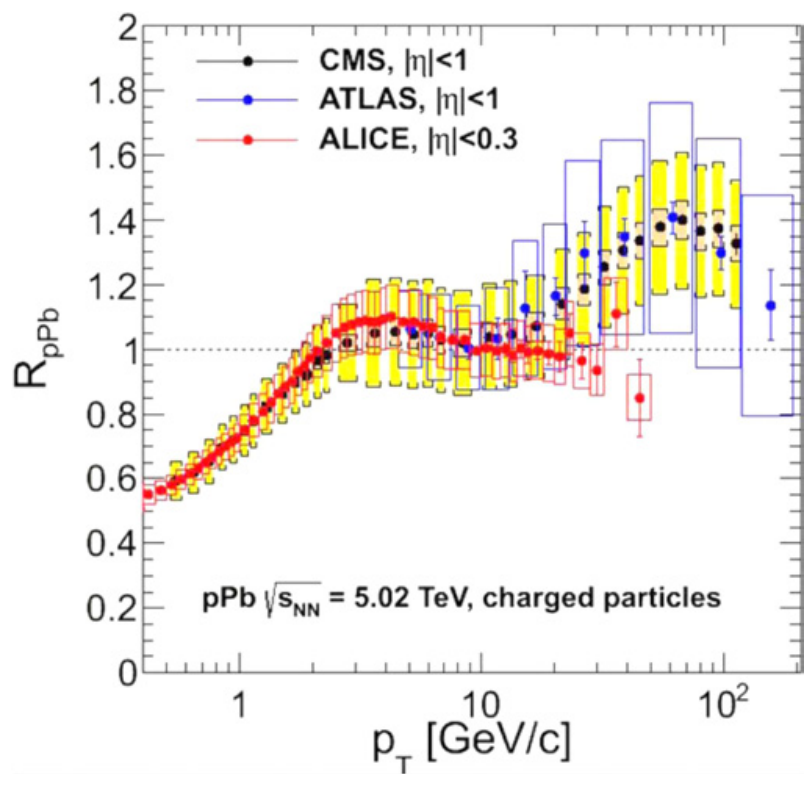

Figure 12. Comparison of the nuclear modification factor $R_{p A}$ of inclusive charged hadrons from ALICE, ATLAS and CMS as shown at QM2014 by Y.-J. Lee [26].

a rise with multiplicity, going from $\mathrm{pp}$ collisions, to $\mathrm{p}-$ $\mathrm{Pb}$ collisions and no further increase in $\mathrm{Pb}-\mathrm{Pb}$ collisions within errors.

Multi-strange particles are of particular interest as their production rate is sensitive to the system size. In Fig. 7 the $\Xi$-to-pion ratio vs. multiplicity is displayed and in Fig. 8 the same for Omega instead of $\Xi$. A rise is observed as for the deuterons-to-proton figure for both ratios. In high multiplicity $\mathrm{p}-\mathrm{Pb}$ collisions similar values as in central $\mathrm{Pb}-\mathrm{Pb}$ collisions are observed for $\Xi^{-}(d s s)$, but not quite for $\Omega^{-}$(sss). Nevertheless, they both reach the prediction from the thermal model reaching $\mathrm{Pb}-\mathrm{Pb}$ collsions. In heavy-ion collisions, chemical equilibrium is 


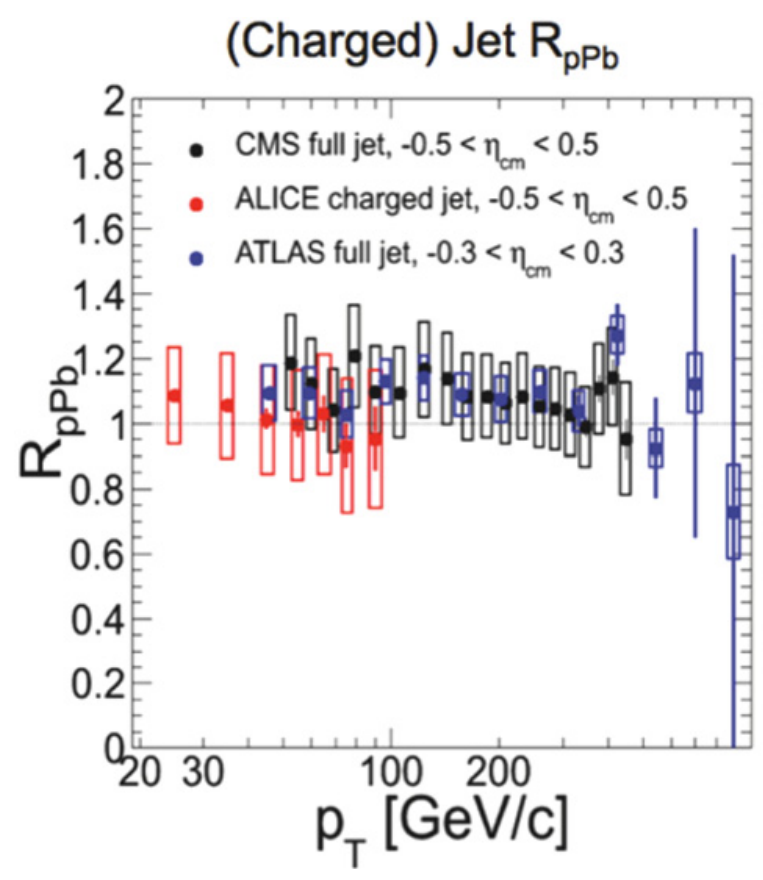

Figure 13. Comparison of the nuclear modification factor $R_{p A}$ of jets from ALICE, ATLAS and CMS as shown at QM2014 by Y.-J. Lee [26].

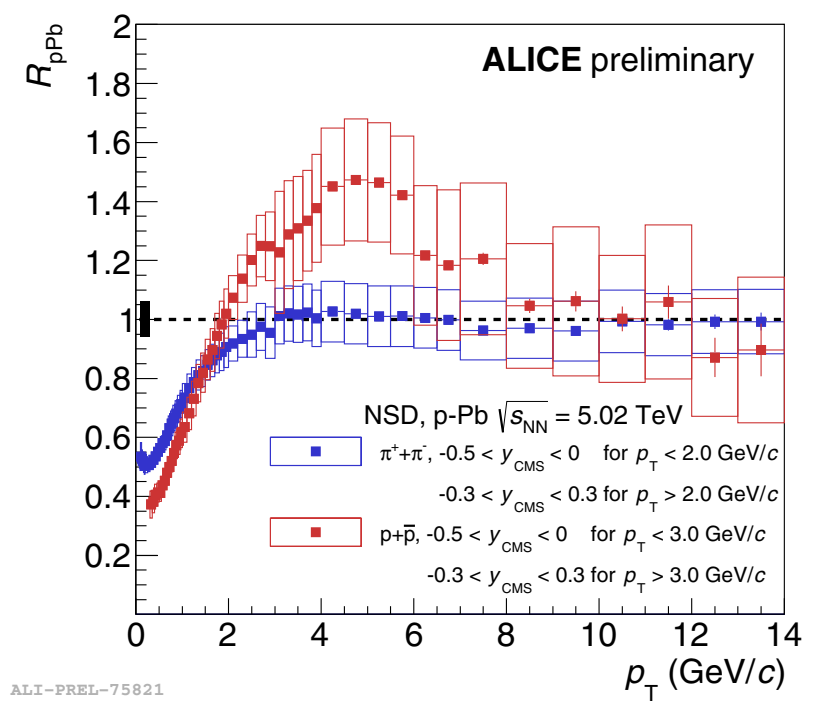

Figure 14. Nuclear modification factor $R_{p A}$ of pions and protons.

typically verified with a thermal fit in which all particle yields are described with the same chemical freeze-out temperature $T_{\mathrm{ch}} \approx 160 \mathrm{MeV}$. For such a fit all measured production yields are used to minimise the Temperature $T_{\text {ch }}$, the Volume $V$ and the baryo-chemical potential $\mu_{B}$ to describe all particles with one set of the three parameters. The volume cancels out if ratios are fitted and at the LHC the baryo-chemical potential is close to zero and as such fixed to null for the current fits. A thermal fit with the three different codes (THERMUS, GSI-Heidelberg and $\mathrm{SHARE}$ ) is shown for $\mathrm{Pb}-\mathrm{Pb}$ in Fig. 9 [13]. The procedure works in 1 st order also in $\mathrm{p}-\mathrm{Pb}$ collisions, however, the $\chi^{2} /$ ndof of the fit is slightly worse: $\approx 5$ instead of $\approx 2$. This is mainly due to the multi-strange particles, which

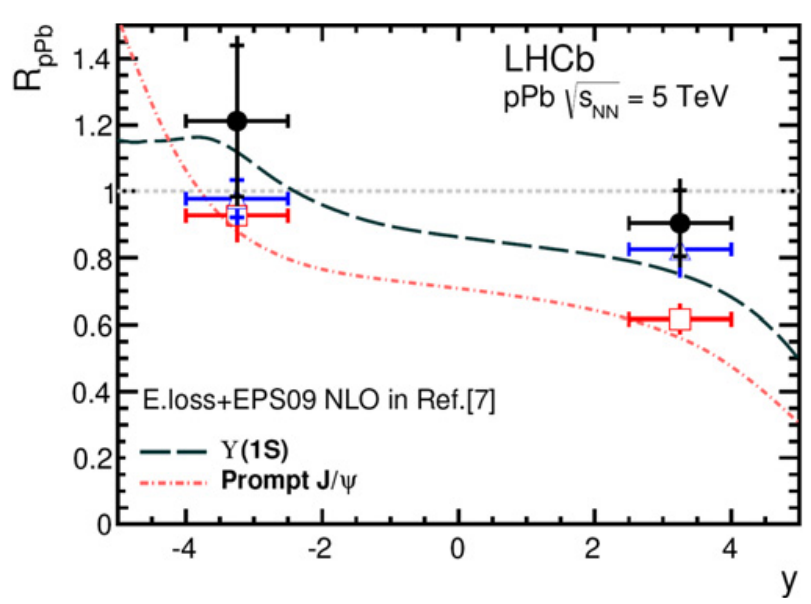

Figure 15. Nuclear modification factor $R_{p A}$ of prompt $\mathrm{J} / \psi$ and $\Upsilon(1 \mathrm{~S})$ from LHCb.

also give some tension to the fit in $\mathrm{Pb}-\mathrm{Pb}$ (opposite to the tension caused there by the protons).

The thermal model predicts the production yield as $\mathrm{d} N_{\mathrm{ch}} / \mathrm{d} y \propto \exp (-m / T)$. In Fig. 10 one sees that the nuclei follow this exponential fall predicted by the model nicely. In other words each added baryon gives a factor of $\approx$ 300 less production yield for the nuclei created in $\mathrm{Pb}-\mathrm{Pb}$ collisions at the LHC.

\section{High $p_{\mathrm{T}}$ and jets}

In relativistic heavy-ion physics, one of the most striking observations is the suppression of particle production at high transverse momenta in heavy-ion compared to $\mathrm{pp}$ collisions [14-19]. The modification of the spectral shape is quantified based on the nuclear modification factor $R_{A A}$, or in case of $\mathrm{p}-\mathrm{A}$ collisions:

$$
R_{p A}=\frac{\mathrm{d} N_{p A} / \mathrm{d} p_{T}}{\left\langle N_{c o l l}\right\rangle \cdot \mathrm{d} N_{p p} / \mathrm{d} p_{T}},
$$

in which the transverse momentum spectrum $\mathrm{d} N_{p A} / \mathrm{d} p_{T}$ obtained in $\mathrm{p}-\mathrm{Pb}$ collisions is divided by the transverse momentum spectrum $\mathrm{d} N_{p p} / \mathrm{d} p_{T}$ obtained in pp collisions which is scaled by the average number of binary nucleonnucleon collisions $N_{\text {coll }}$ in p-Pb collisions. $N_{\text {coll }}$ can be estimated by Glauber calculations and amounts to $6.9 \pm$ 0.6 at LHC energies [20]. For hard processes which can be described by perturbative QCD, a $R_{p A} \approx 1$ indicates that no nuclear effects are present and that this process in $\mathrm{p}-\mathrm{Pb}$ collisions can be described as a superposition of independent proton-nucleon collisions. In general, this behavior is indeed observed, e.g. for the $R_{p A}$ of $\mathrm{D}$ mesons (see Fig. 11) at high $p_{T}$ [21] showing that the suppression effects seen in $A A$ collisions [22] are not due to cold nuclear matter effects, but are final state effects due to the energy lost in the medium. The same observation holds true for the measurement of charged hadrons in a $p_{T}$-region between $10-30 \mathrm{GeV} / c$. At even higher $p_{T}$, however, the CMS and ATLAS collaborations observe an enhancement as shown in Fig. 12. One might think that a possible explanation is given by the modification of the parton distribution functions in the nucleus [23]. 

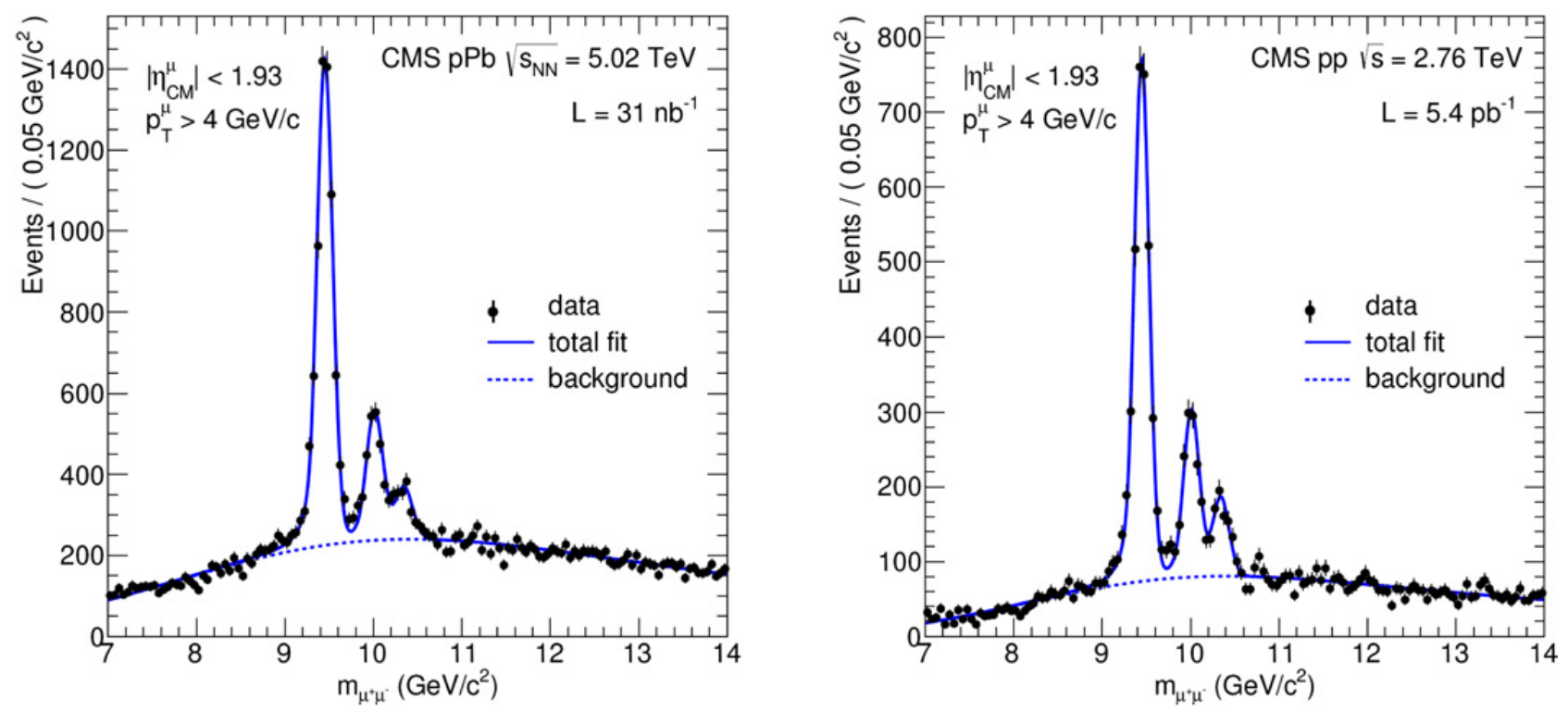

Figure 16. Invariant mass of di-muon pairs for $\mathrm{p}-\mathrm{Pb}$ collisions (left) and $\mathrm{pp}$ collisions (right).
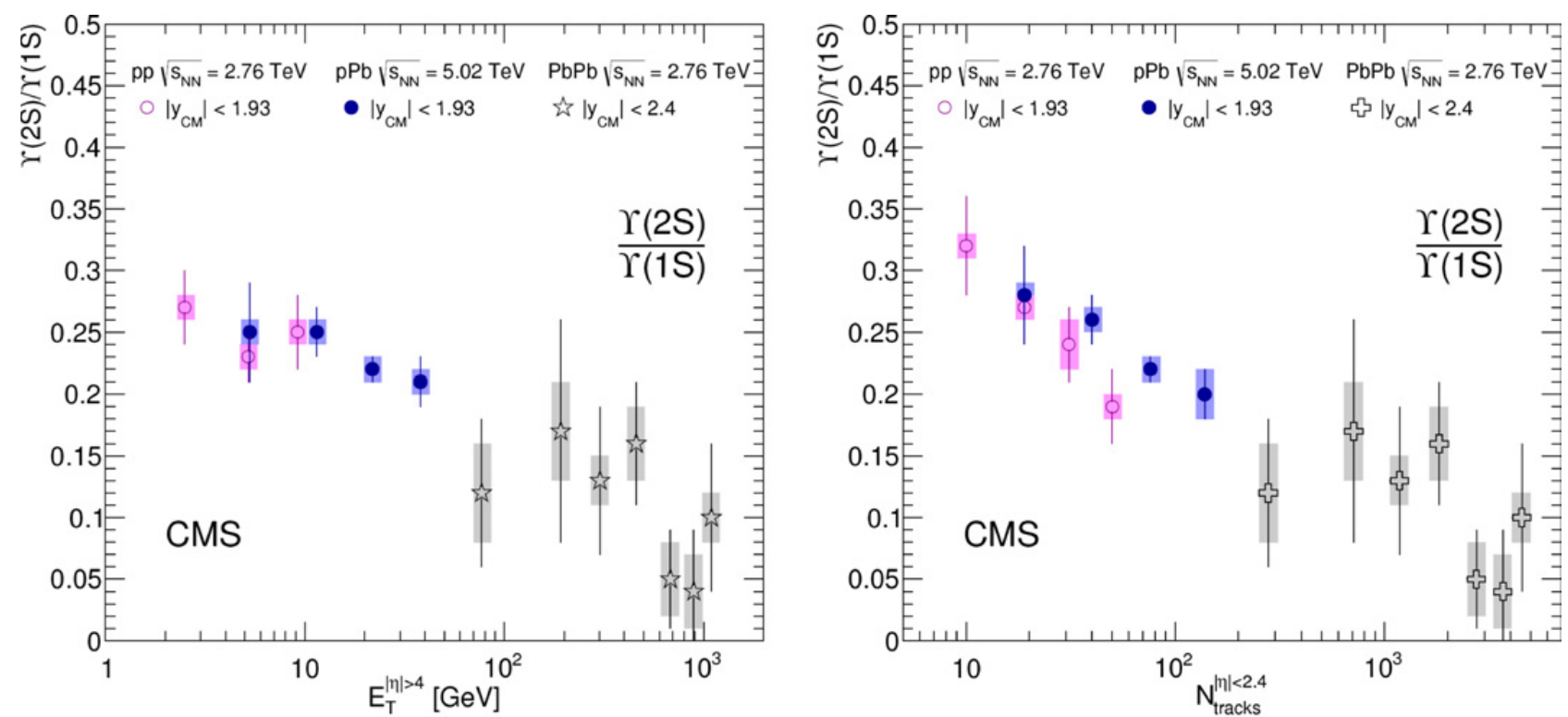

Figure 17. The ratio between $\Upsilon(2 \mathrm{~S})$ and $\Upsilon(1 \mathrm{~S})$ yields measured in $\mathrm{pp}, \mathrm{p}-\mathrm{Pb}$ and $\mathrm{Pb}-\mathrm{Pb}$ collisions over the transverse energy in the event (left panel) and over the number of tracks (right panel). From [30].

However, preliminary estimates show that the expected effects due to anti-shadowing are much smaller than the observed modifications. At the same time, the $R_{p A}$ for charged and full jets is equal to one as also shown in Fig. 13 implying that the jet fragmentation needs to be carefully investigated. From the experimental point of view, it is important to take $\mathrm{pp}$ data at the reference energy in order to verify that the effect is not caused by the interpolation procedure which is used to construct the reference spectrum.

Besides the enhancement at large transverse momenta, the second interesting region in the $R_{p A}$ measurement is the peak region around $3-6 \mathrm{GeV} / c$. It is traditionally interpreted in the context of the Cronin effect as a result of $k_{T}$-broadening [24,25]. As shown in Fig. 14, the height of the peak shows a significant dependence on the particle type. While there is no enhancement for pions, it is rather pronounced for protons indicating that it is consistent with the mass dependent hardening as predicted by the aforementioned radial flow picture.

\section{Heavy flavour and electroweak bosons}

A precise measurement of quarkonia is crucial for the understanding of regeneration effects in $\mathrm{Pb}-\mathrm{Pb}$ collisions which probe de-confinement in $\mathrm{Pb}-\mathrm{Pb}$. In addition, these measurements can help to constrain nuclear PDFs.

In general, nuclear absorption effects are small at the LHC. The precision of the data allows for quantitative comparison with theory. Theoretical predictions based on nuclear shadowing (EPS09 + NLO) are in fair agreement with the $J / \psi$ data shown in Fig. 15. Similarly for models including also partonic energy loss. The same picture 


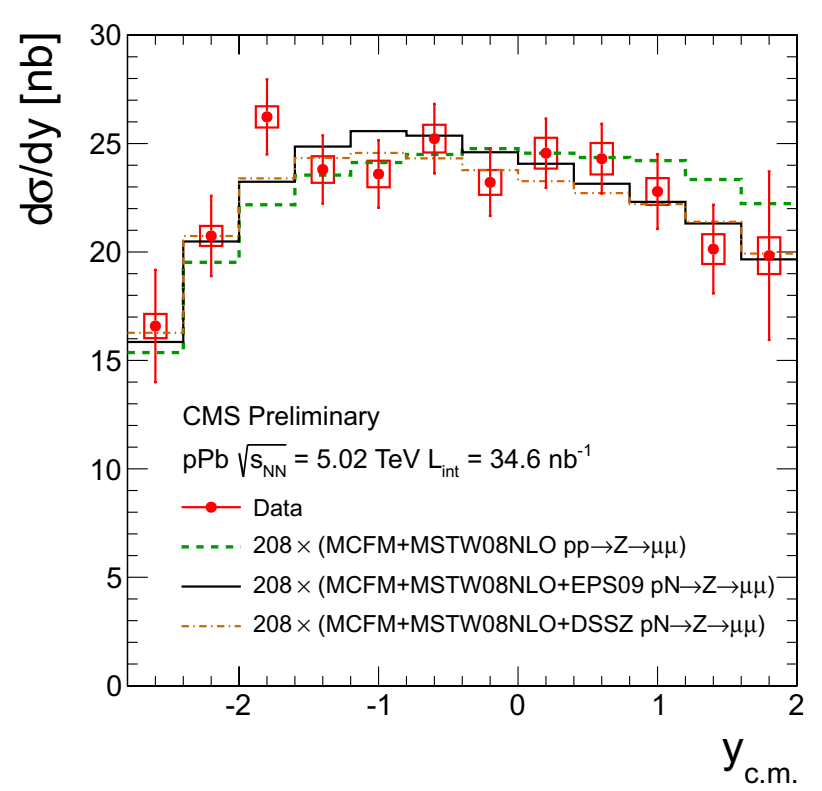

Figure 18. Differential cross section of $Z^{0}$ bosons in $\mathrm{p}-\mathrm{Pb}$ collisions as a function of rapidity compared to predictions from a model for different nPDF sets. All theory predictions are scaled by $\mathrm{A}=208$. The error bars represent the statistical, and the boxes the systematic uncertainties. For details see [32].

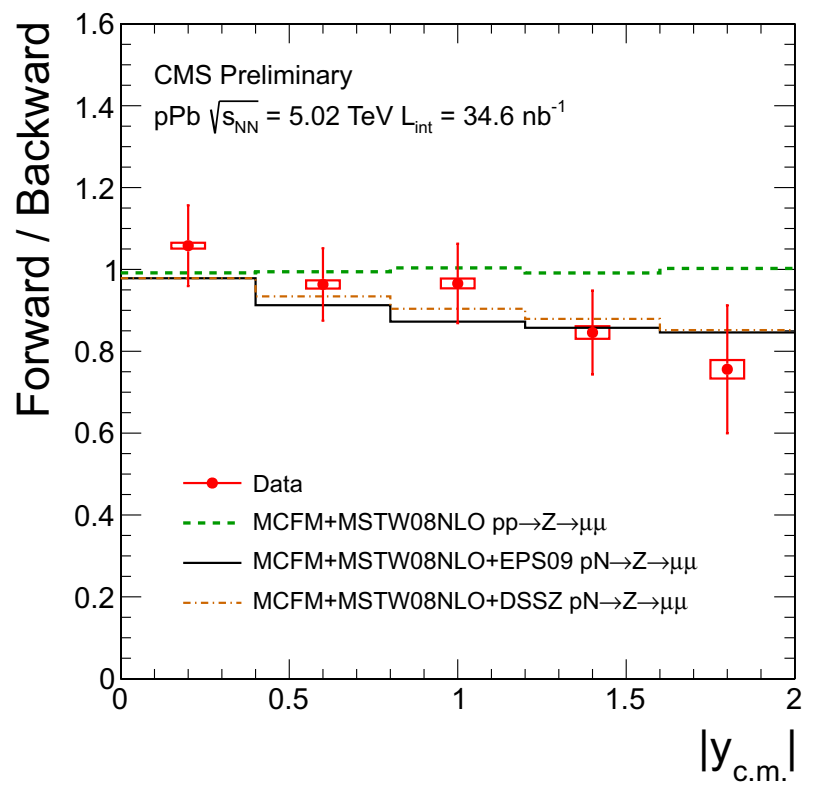

Figure 19. Forward-backward ratio of $Z^{0}$ boson cross section in $\mathrm{p}-\mathrm{Pb}$ collisions as a function of rapidity compared to predictions from a model for different nPDF sets. The error bars represent the statistical, and the boxes the systematic uncertainties. For details see [32].

is true for $\Upsilon$ production [31]. While models predict identical behaviour for $\mathrm{J} / \psi$ and $\psi(2 \mathrm{~s})$, the data shows differences. This is a hint for final state effects, which are unexpected, because charmonia formation time is larger than $c \bar{c}$ crossing time in the nucleus. The suppression might be due to interaction with the (hadronic) medium created in the collision.

Excited $\Upsilon$ states are less suppressed with respect to the ground states in min. bias $\mathrm{p}-\mathrm{Pb}$ collisions than in $\mathrm{Pb}-\mathrm{Pb}$

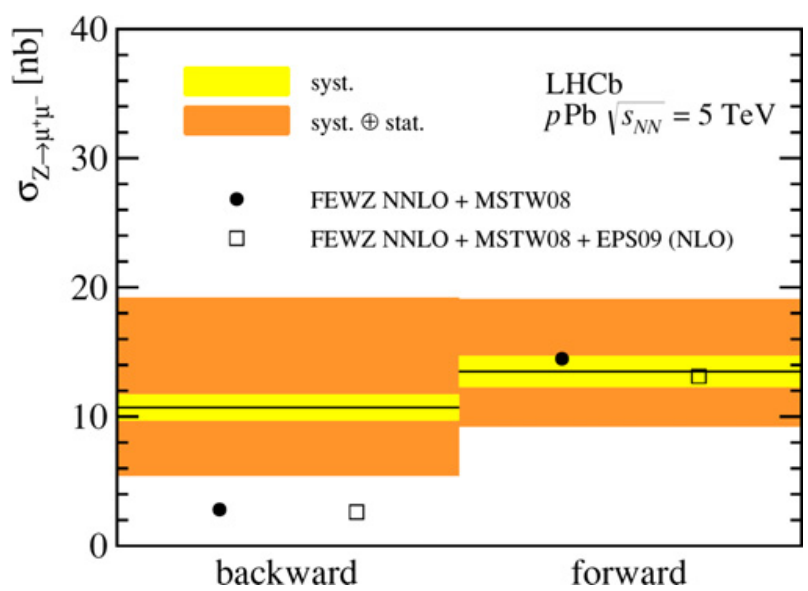

Figure 20. Experimental results and theoretical predictions for the $Z^{0} \rightarrow \mu^{+} \mu^{-}$production cross section, separated for backward and forward direction [33].

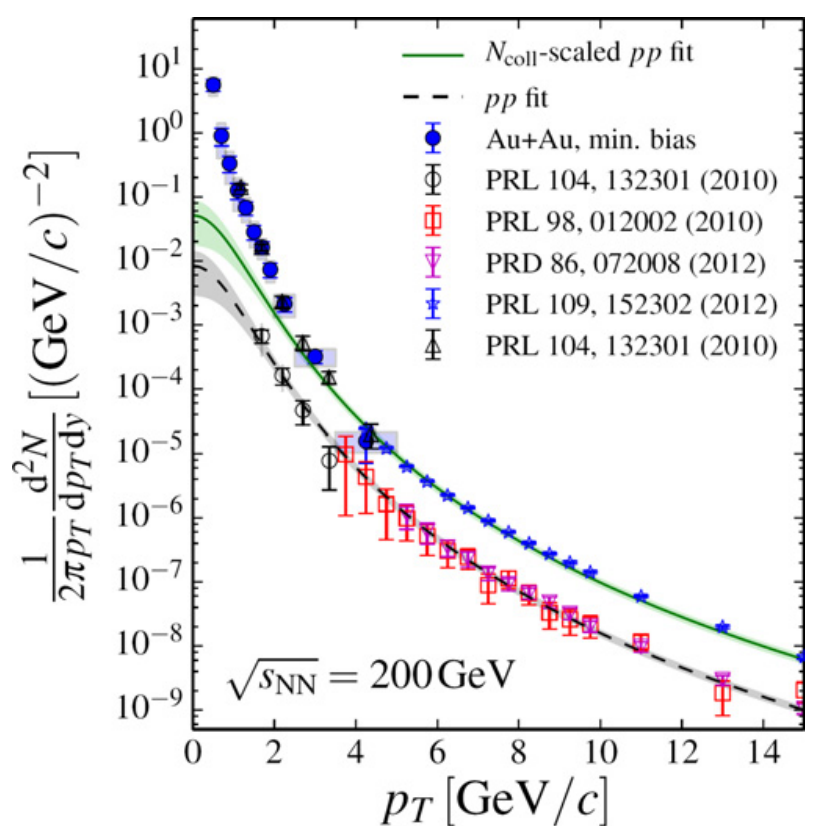

Figure 21. Direct photon $p_{\mathrm{T}}$ spectra for $\mathrm{pp}$ and $\mathrm{Au}-\mathrm{Au}$ collisions. In green the $N_{\text {coll }}$-scaled pp spectrum is shown. The temperature of the quark-gluon plasma is extracted from the difference visible between the spectrum in $\mathrm{Au}-\mathrm{Au}$ and the $N_{\text {coll }}$-scaled pp spectrum. For details see [34].

collisions. The invariant mass of di-muon pairs is shown in Fig. 16. However, the suppression of excited states seems to vary with the event multiplicity (same in pp) as shown in Fig. 17. It is an open question if excited states add multiplicity (event selection bias) or if the activity suppresses excited states (as in $\mathrm{Pb}-\mathrm{Pb}$ collisions).

A completely new field opened at the LHC with the accessibility of the measurement of the bosons of the weak interaction, which was not possible at the RHIC energies. For instance, around $2200 Z^{0}$ are measured at CMS in the di-muon channel (similar values for ATLAS) in $\mathrm{p}-\mathrm{Pb}$ collisions. There are also results from $\mathrm{LHCb}$, but with much smaller statistics (around 15 candidates). The cross-section measured with CMS (see Fig. 18) is in fair agreement with the predictions but the ratio between forward and backward direction shows some tension 


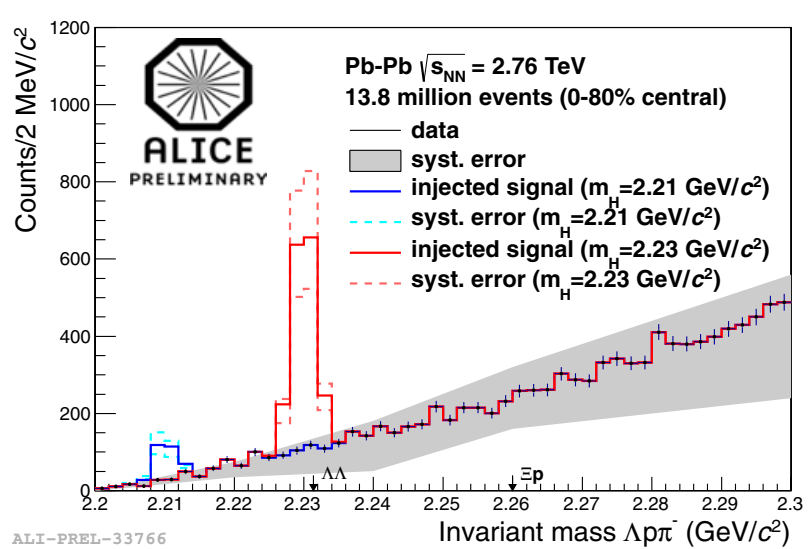

Figure 22. Invariant mass distribution of $\Lambda \mathrm{p} \pi^{-}$measured with ALICE in $\mathrm{Pb}-\mathrm{Pb}$ collisions as black markers. In red is a signal from Monte Carlo simulations assuming a binding energy of $1 \mathrm{MeV}$ and in blue a simulated signal for a $20 \mathrm{MeV}$ bound $\mathrm{H}-$ dibaryon. The gray band shows the systematic uncertainty of the data.

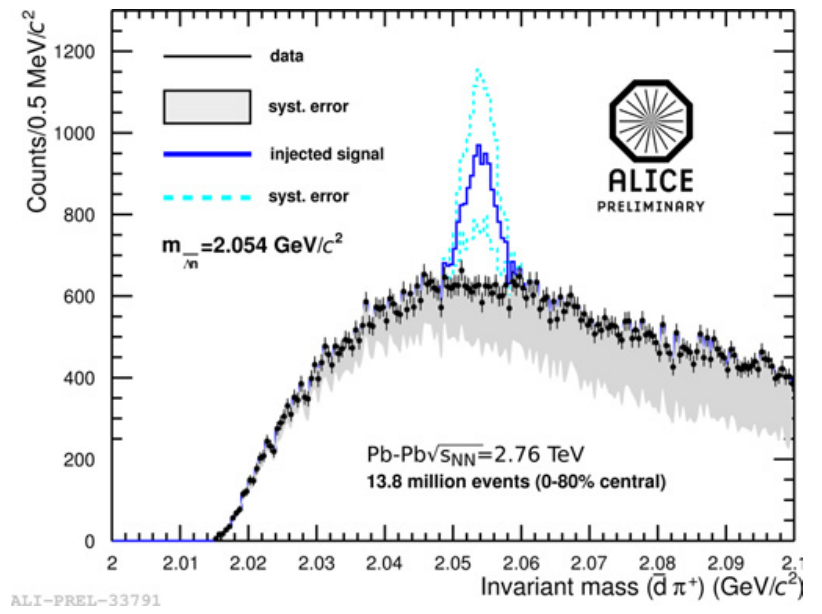

Figure 23. Invariant mass of $\bar{d} \pi$ measured by ALICE in $\mathrm{Pb}-$ $\mathrm{Pb}$ collisions in black and in gray the corresponding systematic uncertainty. In blue a simulated signal of the discussed $\Lambda \mathrm{n}$ bound state is visualised.

compared with models, as visible in Fig. 19. LHCb has observed the same tension as displayed in Fig. 20. This hints of an asymmetry in the forward-backward direction might help to contrain nuclear PDFs. Similar studies have been started for the $W^{+}$and the $W^{-}$.

Another interesting probe are direct photons, which have to be separated from the photons coming from decays. From the measurements of direct photon $p_{\mathrm{T}}$ spectra (see Fig. 21), when pp and $\mathrm{Au}-\mathrm{Au}$ (or $\mathrm{Pb}-\mathrm{Pb}$ ) are compared, one can extract the temperature of the created medium in heavy-ion collisions. The values at RHIC and LHC are above $\approx 250 \mathrm{MeV}$.

\section{Search for exotic objects}

The energies reached in relativistic heavy-ion collisions lead to the abundant production of rare probes, as briefly discussed for the electroweak bosons in Sect. 4. This means also particles which are more exotic should be possible to be created in these collisions. We focus here on three examples, first the H-dibaryon (a hexaquark state,

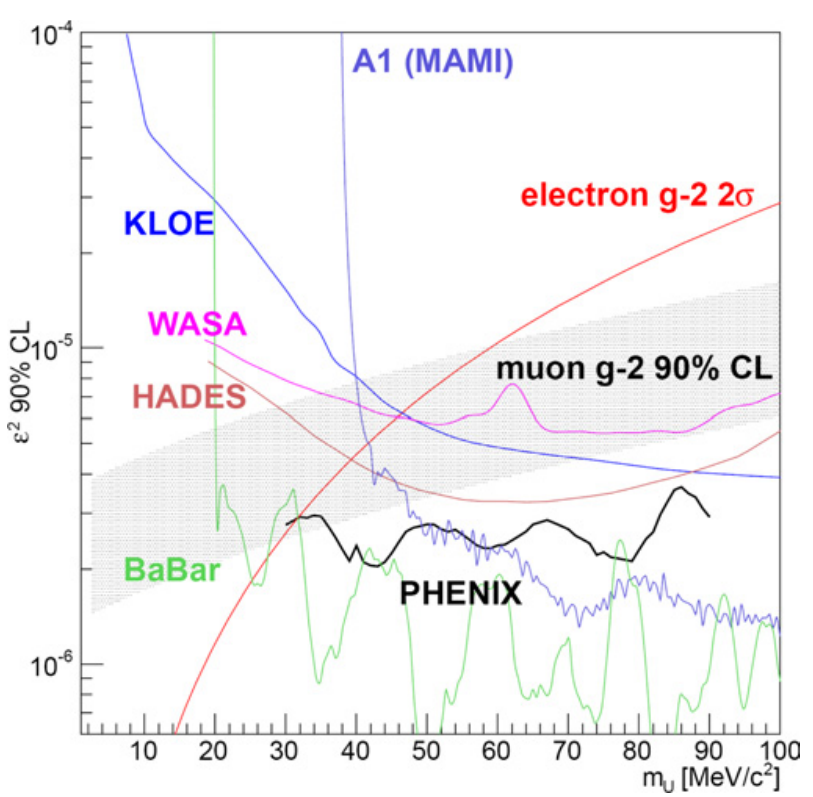

Figure 24. Comparison of different dark photon limits at $90 \%$ confidence level and the possible band from the measured muon anomalous magnetic moment $(g-2)_{\mu}$. Taken from [35].

composed of uuddss quarks) in its possible weak decay mode $\mathrm{H}$-dibaryon $\rightarrow \Lambda \mathrm{p} \pi^{-}$. Its measured invariant mass is shown in Fig. 22 on top of its mass the expected signal is plotted for a slightly bound $\mathrm{H}$-dibaryon ( $1 \mathrm{MeV}$ binding energy) and for a stronger bound H-dibaryon (20 MeV binding energy). For the expected signal Monte Carlo was used to estimate the efficiency and the expected yield was determined by using the thermal model (introduced in Sect. 2.2). Since no signal was observed, upper limits have been determined.

A second exotic object is the hypothetical bound state of a $\Lambda$ and a neutron, which could decay into a deuteron and a pion. In Fig. 23 its invariant mass is displayed. No signal was observed and the peak shown is the expectation from thermal model. Also here upper limits have been set. For more information see [10] and references therein.

The last exotic object whose search should be shortly discussed here is the so-called dark photon $U$. Its existence could explain the discrepancy between the measured muon anomalous magnetic moment $(g-2)_{\mu}$ and the standard model prediction. It is an additional U(1) gauge boson, which is weakly coupled to ordinary photons. The mixing with the ordinary photons is expected to be visible in the Dalitz-decay of the $\pi^{0}$ and the $\eta$. Also here no signal is observed and upper limits over a mass range is set. The currently observed upper limt by the PHENIX Collaboration is shown in Fig. 24, compared with the limit of other experiments (HADES is also a heavy-ion experiment hosted at GSI and thus at lower collision energies as discussed in these proceedings).

\section{Summary and conclusion}

There are a lot of interesting physics results from $\mathrm{A}-\mathrm{A}$ and in particular $\mathrm{p}-\mathrm{A} / \mathrm{d}-\mathrm{A}$ collsion data, which have been shown here. The most prominent are $\mathrm{Pb}-\mathrm{Pb} / \mathrm{Au}-\mathrm{Au}$ like features which are observed for the bulk of the produced 
particles at low $p_{\mathrm{T}}: v_{2}$ and radial flow show a collective behaviour which speaks for possible kinetic equillibrium, whereas the thermal fits attribute a hint of chemical thermal equilibrium. Further, there is no indication of quenching at high $p_{\mathrm{T}}$ in $\mathrm{p}-\mathrm{Pb}$ collisions for charged hadrons, jets, open charm, heavy flavour, electrons and muons. However, CMS and ATLAS observe a yet unexplained enhancement at $p_{\mathrm{T}}$. The shown quarkonia measurements provide an essential baseline for the understanding of the $\mathrm{Pb}-\mathrm{Pb}$ results. On the other hand, electroweak bosons can help to constrain nPDFs and centrality estimators. For the discussed searches of exotic objects significant upper limits have been set. Altoghether this means we have very interesting times ahead of us!

I would like to thank the ALICE, ATLAS, CMS, LHCb, PHENIX and STAR Collaborations for the support and the provided material.

\section{References}

[1] K. Aamodt et al. (ALICE Collaboration), Phys. Rev. Lett. 105, 252301 (2010)

[2] B. Abelev et al. (ALICE Collaboration), Phys. Lett. B 728, 25 (2014)

[3] K. Aamodt et al. (ALICE Collaboration), Eur. Phys. J. C 68, 345 (2010)

[4] P. Braun-Munzinger, J. Stachel, C. Wetterich, Phys. Lett. B 596, 61 (2004)

[5] U. Heinz, arXiv:hep-ph/0407360v1

[6] S. Chatrchyan et al. (CMS Collaboration), JHEP 09, 091 (2010)

[7] S. Chatrchyan et al. (CMS Collaboration), Phys. Lett. B 718, 795 (2013)

[8] B. Abelev et al. (ALICE Collaboration), Phys. Lett. B 726, 164 (2013)

[9] B. Abelev et al. (ALICE Collaboration), arXiv:1405.4632v1 [nucl-ex]

[10] N. Martin (for the ALICE Collaboration), Nucl. Phys. A 931, 1103 (2014)

[11] C. Andrei (for the ALICE Collaboration), Nucl. Phys. A 931, 888 (2014)

[12] D. Alexandre (for the ALICE Collaboration), Nucl. Phys. A 931, 1093 (2014)
[13] M. Floris, Nucl. Phys. A 931, 103 (2014)

[14] K. Aamodt et al. (ALICE Collaboration), Phys. Lett. B 696, 30 (2011)

[15] S. Chatrchyan et al. (CMS Collaboration), Eur. Phys. J. C 72, 1945 (2012)

[16] I. Arsene et al. (BRAHMS Collaboration), Nucl. Phys. A 757, 1 (2014)

[17] B.B. Back et al. (PHOBOS Collaboration), Nucl. Phys. A 757, 28 (2014)

[18] J. Adams et al. (STAR Collaboration), Nucl. Phys. A 757, 102 (2014)

[19] K. Adcox et al. (PHENIX Collaboration), Nucl. Phys. A 757, 184 (2014)

[20] A. Toia (for the ALICE Collaboration), Nucl. Phys. A 931, 315 (2014)

[21] B. Abelev et al. (ALICE Collaboration), arXiv:1405.3452v1 [nucl-ex]

[22] B. Abelev et al. (ALICE Collaboration), JHEP 9, 112 (2012)

[23] K. J. Eskola, H. Paukkunen, C. A. Salgado, JHEP 04, 65 (2009)

[24] J.W. Cronin et al., Phys. Rev. D 11, 3105 (1975)

[25] S.S. Adler et al. (PHENIX Collaboration), Phys. Rev. C 74, 024904 (2006)

[26] Y.-J. Lee, talk at the XXIV. International Conference on Ultrarelativistic Nucleus-Nucleus Collisions (QM2014), Darmstadt, 2014

[27] A. Sickles, Nucl. Phys. A 931, 103 (2014)

[28] A. Adare et al. (PHENIX Collaboration), Phys. Rev. C 88, 024906 (2013)

[29] B. Abelev et al. (ALICE Collaboration), arXiv:1405.3796v1 [nucl-ex]

[30] S. Chatrchyan et al. (CMS Collaboration), JHEP 04, 103 (2014)

[31] R. Aaij et al. (LHCb Collaboration), JHEP 07, 094 (2014)

[32] S. Chatrchyan et al. (CMS Collaboration), CMSHIN-14-003

[33] R. Aaij et al. (LHCb Collaboration), JHEP 09, 030 (2014)

[34] A. Adare et al. (PHENIX Collaboration), arXiv:1405.3940v1 [nucl-ex]

[35] A. Adare et al. (PHENIX Collaboration), arXiv:1409.0851v2 [nucl-ex] 